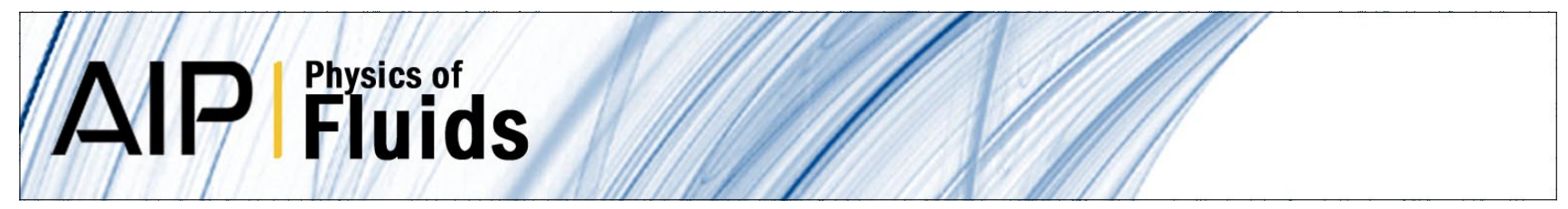

\title{
An exploration of the wake three dimensionalities caused by a local discontinuity in cylinder diameter
}

C. G. Lewis and M. Gharib

Citation: Phys. Fluids A 4, 104 (1992); doi: 10.1063/1.858489

View online: http://dx.doi.org/10.1063/1.858489

View Table of Contents: http://pof.aip.org/resource/1/PFADEB/v4/i1

Published by the AIP Publishing LLC.

\section{Additional information on Phys. Fluids A}

Journal Homepage: http://pof.aip.org/

Journal Information: http://pof.aip.org/about/about_the_journal

Top downloads: http://pof.aip.org/features/most_downloaded

Information for Authors: http://pof.aip.org/authors

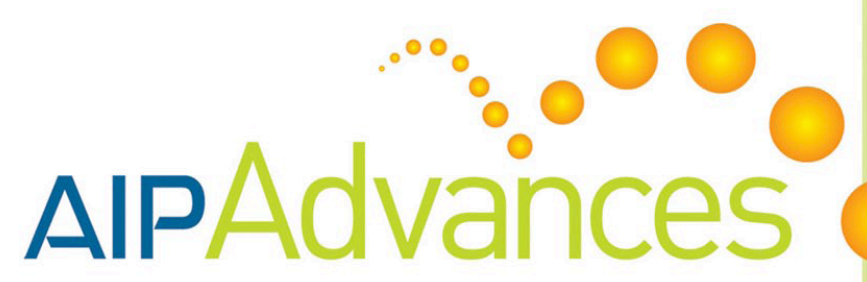

Submit Now
Explore AIP's new open-access journal

Article-level metrics now available

- Join the conversation! Rate \& comment on articles 


\title{
An exploration of the wake three dimensionalities caused by a local discontinuity in cylinder diameter
}

\author{
C. G. Lewis and M. Gharib \\ University of California at San Diego, Mail Code 0411, San Diego, California 92093
}

(Received 14 November 1990; accepted 19 September 1991)

\begin{abstract}
The wake three-dimensionality caused by a local discontinuity in cylinder diameter (a stepped cylinder) was studied. Changes in vortex shedding frequency caused by the discontinuity took place in either a direct or an indirect mode, depending on the diameter ratio and the Reynolds number. The roles of oblique shedding and frequency modulation of vortex shedding were studied. The physical change in vortex shedding frequency occurred differently for each mode. A wavelet analysis is used to explore frequency modulations in the wake. The relationship between vortex linkages, frequency changes, secondary instability, and these two modes is discussed.
\end{abstract}

\section{INTRODUCTION}

Although the circular cylinder is a commonly used model for the study of two-dimensional separated flows, it is difficult to prevent three-dimensional phenomena from evolving in such flows. It is now believed that unwanted three-dimensionality may have been the source of discrepancies in many reported results on circular cylinder wakes. In this regard, attempts have been made to understand the physical nature of the coupling between the two- and threedimensional processes in the flow.

For example, Gaster investigated the nature of three dimensionality in the wake of a conc ${ }^{1}$ and a tapered cylinder. ${ }^{2}$ In the cone study, Gaster finds low-frequency modulated shedding throughout the wake. In the tapered cylinder study, he reports that shedding along the span of the cylinder breaks into a number of discrete cells. Within each cell, the shedding is regular and periodic, but at the cell boundary there is a beating of the neighboring cell frequencies. Apparently, the difference in the two results is due to the higher taper ratio of the cone. Gaster also shows that very similar behavior is seen in the cylinder wake when the incoming flow is nonuniform. Studies on cylinders in shear flows ${ }^{3,4}$ show similar wake behavior, in which the shedding breaks into cells of constant frequency. The aforementioned experiments indicate that wake behavior is very sensitive to taper or shear ratio.

The importance of end effects on cylinder wakes has been recognized for some time. Stansby ${ }^{5}$ studied the effect of endplates on the base pressure coefficients of cylinders. Gerich and Eckelmann ${ }^{6}$ investigate the vortex shedding frequencies in the vicinity of base plates and cylinder free ends, and find that shedding frequency is constant and of lower frequency in the end region. At the border of the end region is a shedding discontinuity, and a beating of neighboring frequencies exists. Williamson ${ }^{7}$ finds that manipulation of base plates at cylinder ends alters the frequency and angle of vortex shedding in the cylinder wake. Konig et al. ${ }^{8}$ find that changes in cylinder end conditions affect not only frequency and angle of vortex shedding, but also alter the number and location of the shedding discontinuities.
All of the three dimensionalities caused by diameter taper, velocity shear, and end conditions (regions of local velocity nonuniformity), are similar in behavior. In all of these flows, there is a spanwise variation of Strouhal frequency that is tied in with the three-dimensional vorticity dynamics. The wake behavior must accommodate the rate of change of the shedding frequency along the span of the model. For lower rates of change, cells of constant frequency shedding appear. Between these regions, the neighboring vortex structures are forced to find suitable terminations or linkages. A model for the linkages of vortices in these regions is suggested by Gerrard. ${ }^{9}$ Williamson ${ }^{7}$ also observes spanwise discontinuity in the frequency of vortex shedding and proposes a possible linkage model.

In the mixing layer, Browand investigated spanwise discontinuity in the roll-up process of vortices, both as it occurs naturally, ${ }^{10,11}$ and as a result of temporal/local forcing. ${ }^{11,12}$ For these cases, a two-dimensional (2-D) plus time nonlinear evolution equation model is suggested by Huerre ${ }^{13}$ (for parallel free shear flows that are invariant under space translations and Galilean transformations). A secondary instability, coupling pattern deformation and a large-scale advection, is found to describe the resulting complex vortex linkages (or defects).

There have been a number of studies that focus on the stepped cylinder geometry. Ko et al. ${ }^{14}$ studied the pressure fluctuations in the wake of a stepped cylinder with a 2:1 diameter ratio, at high Reynolds numbers. Their measurements focus on a region within three diameters of the diameter discontinuity. The same geometry with the addition of endplates, and an aspect ratio of 10, was studied by Ko and Chan ${ }^{15}$ at high Reynolds number. They investigate vortex coherence and phase in the localized region behind the diameter discontinuity, and compare it to the results generated by two-dimensional models of the same diameter and aspect ratio.

In summary, three-dimensionality is typically found in regions of spanwise variation in either velocity or diameter (for the cylinder). These variations of the two-dimensional cylinder flow serve as basic simplified models for many important and realistic flows of interest. Our interest is in the 
study of three-dimensionality in wakes, and in the vortex linkages. The intent of this study is to simplify the more complex shedding phenomena induced by spatial variations by using a single jump in cylinder diameter, a stepped cylinder. We will study the laminar shedding range, using largeaspect-ratio models.

\section{APPARATUS, EXPERIMENTAL METHODS, DATA PROCESSING}

\section{A. Methods and apparatus}

The experiments were carried out in a closed-circuit wind tunnel with a working section of $61 \times 61 \mathrm{~cm}(24 \times 24$ in.). The background turbulence level of the facility was less than $0.03 \%$. The tunnel wall boundary layers were $2.5 \mathrm{~cm}$ at the lowest velocity.

Stepped cylinders were made of stainless steel hypodermic tubing tightly fit over a music wire core. Care was taken to avoid cylinder vibration. ${ }^{16}$ The experimental setup is shown in Fig. 1. The cylinder model diameters $d_{L}$ and $d_{S}$, diameter ratio $r$, and aspect ratios $A r=L / d_{L}$, $A r_{L}=L_{L} / d_{L}$, and $A r_{S}=L_{S} / d_{S}$ are given in Table $\mathrm{I}$.

Velocity measurements were taken using standard hotwire anemometry techniques. Mini hot films were used to increase spatial resolution of the measurements and were oriented parallel to the cylinders. Velocity measurements were taken with a Masscomp data acquisition computer system.

Flow visualization studies were conducted using a smoke wire and recorded with a Cid Tek camera on highresolution videotape. ${ }^{17}$ Oblique angle measurements were averaged from several videotape hard copies.

Preliminary experiments were conducted with endplates, but a comparison to cases without endplates indicated that our aspect ratios were more than sufficient to warrant leaving them off. Control cylinders ${ }^{18}$ were also employed to create symmetric boundary conditions. The behavior of shedding angle with Reynolds number was virtually unchanged. The effect of the step was so strong that the need for symmetric boundary conditions was found to be unnecessary at these high-aspect ratios.

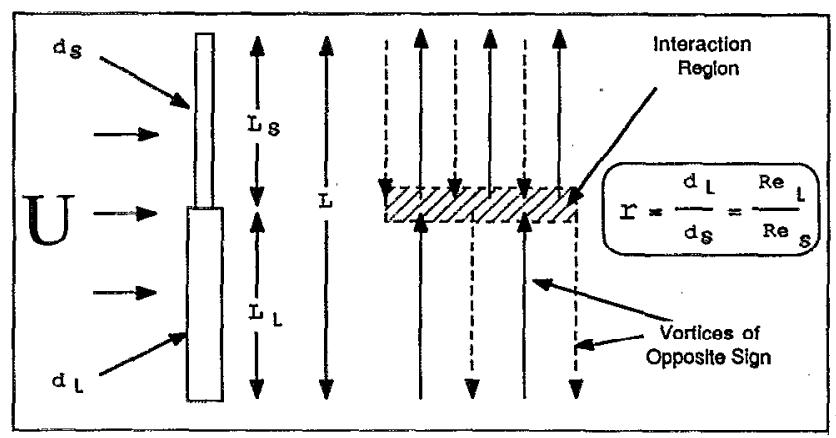

FIG. 1. Sketch of the experimental setup.
TABLE I. Cylinder model diameters and aspect ratios.

\begin{tabular}{llllll}
\hline $\begin{array}{l}\text { Diameter } \\
\text { ratio }(r)\end{array}$ & $\begin{array}{c}d_{L} \\
(\mathrm{~cm})\end{array}$ & $\begin{array}{c}d_{S} \\
(\mathrm{~cm})\end{array}$ & $A r_{L}$ & $A r_{S}$ & $\begin{array}{c}A r(\text { based } \\
\left.\text { on } d_{L}\right)\end{array}$ \\
\hline 1.14 & 0.2413 & 0.2108 & 126 & 145 & 252 \\
1.27 & 0.3048 & 0.2413 & 100 & 126 & 200 \\
1.34 & 0.2108 & 0.1575 & 145 & 194 & 290 \\
1.53 & 0.2413 & 0.1575 & 126 & 194 & 252 \\
1.76 & 0.2769 & 0.1575 & 110 & 194 & 220 \\
\hline
\end{tabular}

\section{B. Data analysis}

Because of the highly transient nature of the flow in the interaction region, a local frequency analysis technique (the wavelet transform ${ }^{19}$ ) was used. The wavelet transform provides a local time frequency analysis at each scale analyzed. ${ }^{20}$ The wavelet coefficients were normalized to range between +1 and -1 . For a periodic signal, the Mexican hat wavelet transform at the proper scale yields normalized coefficients that vary periodically from +1 to -1 . A wavelet coefficient is similar to a correlation coefficient, but gives local information over single wavelengths. For a periodic signal, the phase is constant at the maxima. If a signal is amplitude modulated, the wavelet coefficients will also be modulated. In the absence of a signal at the scale of the analyzing wavelet, the coefficients will go to zero, so gaps in the periodicity of a signal are easily found. Variations in the time behavior of vortex structures can be studied using these properties.

We implemented a local frequency analysis that uses the output of the wavelet analysis at the scale of the vortex structures. Points of equal phase for the retained structures were found (the maxima were used), and the local frequency was defined to be the inverse of the time between these points. The output of the local frequency analysis quantizes the temporal spacing of vortex structures in a simple manner. Structures having a wavelet coefficient of less than 0.4 at the maxima are removed from the analysis. (Some cutoff value is necessary. We chose to remove waveforms whose amplitudes were less than half of the maximum.) The weak structures (those with the normalized wavelet coefficient of less than 0.4) will yield low local frequencies, indicating either gaps or regions of weak vortex structures in the vortex street.

Frequency modulation of vortex shedding is manifested by variation of the local frequency values. The local frequency values are a quantization of the actual frequency as it varies in time. Amplitude modulation, on the other hand, will yield values of constant local frequency, except in the vicinity of the zeros of the modulation envelope. In these regions, a subharmonic local frequency will be found that corresponds to the number of missed or weak vortex structures in the analysis.

[A similar analysis could be performed on low-noise, laminar data using a narrow finite impulse response (FIR) bandpass filter instead of the wavelet transform. The local frequency analysis could then be implemented on the filtered data. However, the technique we have used is robust and 
avoids the iterative procedure necessary in creating a good narrow bandpass filter, so it is easier to use. Furthermore, the wavelet method could be applied to turbulent data, or data with a low signal-to-noise ratio.]

\section{EXPERIMENTAL RESULTS}

A local discontinuity in cylinder diameter causes a discontinuity in shedding frequency to occur at the interface region of the wakes (see Fig. 1). Based on local frequency analysis, FFT's, and flow visualization, two distinct types of wake behavior were identified, which we will call the direct and indirect modes of interaction.

The appearance of these different modes is a function of the diameter ratio and Reynolds number $(\mathrm{Re})$. The interaction region behavior can be divided into three categories, based on $r$ :

$r<1.25$; direct mode dominates,

$1.25<r<1.55$; transitional (mode is a function of $\mathrm{Re}$ ),

\section{$1.55<r$; indirect mode dominates.}

We will first describe the general characteristics of the two different modes. The indirect mode was complicated by temporal and spatial variation as the Reynolds number increased. So, the indirect mode is first discussed for the low Reynolds number case when the small diameter wake is not shedding. This simple case had the same features as higher Reynolds number cases, but was periodic in time, without the variation seen at higher Reynolds numbers. We will then discuss the three categories listed above, and the role of Reynolds number. The range of Reynolds numbers under investigation was $35<\operatorname{Re}<200$.

The subscripts $S$ and $L$ are used to distinguish between small-diameter and large-diameter variables, respectively.

\section{A. Modes of the interaction region}

\section{Direct mode}

When the direct mode occured, two distinct shedding frequencies were found, which interacted directly with one another in the interaction region (Fig. 1). This region is a narrow zone of quasiperiodic frequency interaction. The vortex lines are interrupted along a plane, which we call the interface. Figure 2(a) shows a typical flow visualization of this mode. Vortices connect across the interface when they are in phase (or nearly so). As they become more out of phase, the vortices form linkages to one another on the same side of the interface. As a result, a hole is formed in the vortex pattern, representing an absence of vortex tubes (at the interface). Figure 2(b) is a schematic of the vortex linkages that are seen in the photograph. These are typical for the direct mode. As the ratio of the shedding frequencies varies, the ratio of the number of vortices in one beat cycle will also change. There are extra vortex tubes on the smalldiameter side. In the simplest case, an extra vortex connects to the vortex of opposite sign that follows it (on the same side of the vortex street). This connection can be observed in flow visualization as a half-loop. At least one half-loop is observed per beat cycle. At the interface, when the hot-film probe is at the edge of the wake, these half-loops are missing

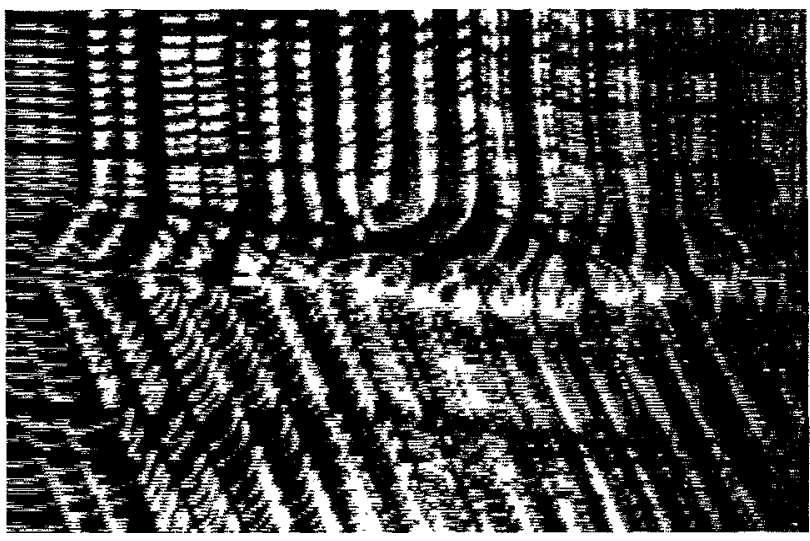

(a)

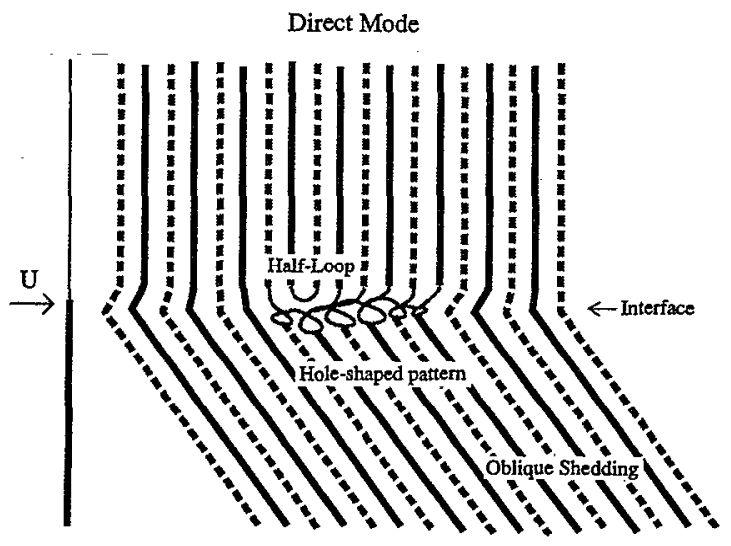

(b)

FIG. 2. (a) Flow visualization at Reynolds number $76 / 57$ for the 1.34 diameter ratio. This is the direct mode. (b) Sketch of (a) showing the vortex lines and the vortex linkages as we interpret them from flow visualization and data analysis. The fcatures discussed in the text are labeled.

or are weak vortices, and give subharmonics of the shedding frequency in the local frequency analysis. Sequences of halfloops often form, yielding lower subharmonics. Weak vortex linkages can, at times, be observed to occur across the edges of the hole region. These are zeroed out by the cutoff chosen in the local frequency analysis. Our analysis gives the local frequency of the strong vortices that result from the connections that span the interface.

\section{Indirect mode}

When the indirect mode appeared, a third frequency $f_{3}$ was found near the interface in the large-diameter wake. This region is called the modulated zone, and acts as a buffer zone between the two wake frequencies $f_{L}$ and $f_{S}$, which no longer interact directly. The frequency $f_{3}$ was always lower than the two wake shedding frequencies $f_{L}$ and $f_{S}$. It was not a subharmonic or the result of a quasiperiodic interaction of $f_{L}$ and $f_{S}$.

This mode has a distinct appearance [Fig. 3(a) ]. Figure 3 (b) is a sketch showing the features of this mode. Vortex linkages in the interface zone occur along a line that is nearly 


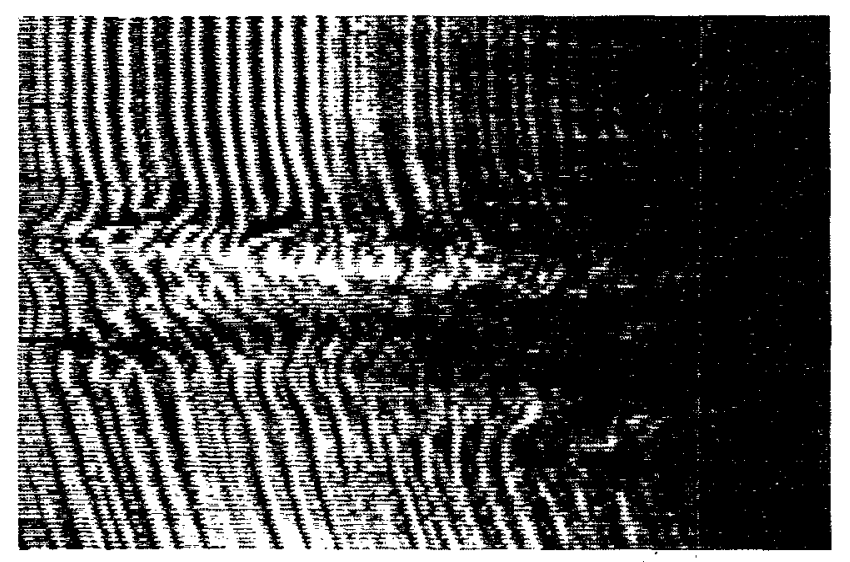

(a)

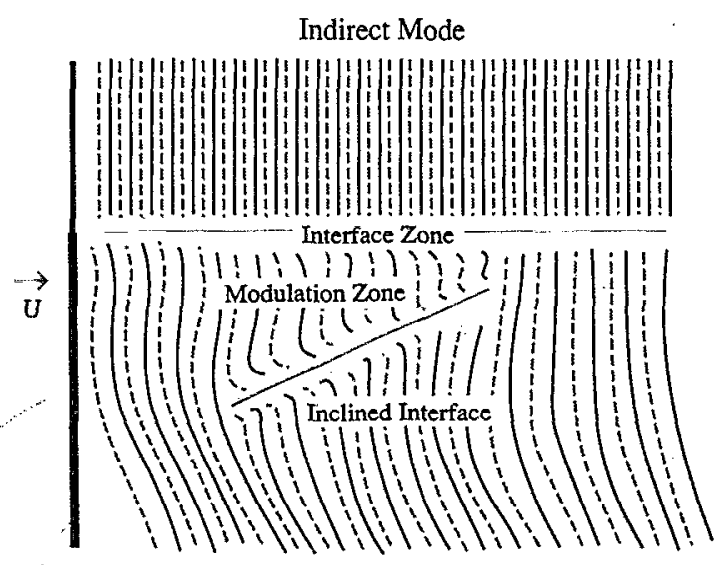

(b)

FIG. 3. (a) Flow visualization of the indirect mode for the Reynolds number $99 / 56$ case for the 1.76 diameter ratio. (b) Sketch of the indirect mode, showing the general features of this mode, as discussed in the text. The sketch is not intended to reflect the precise conditions of (a).

perpendicular to the axis of the cylinders. The interface zone is a region of frequency interaction between $f_{S}$ and $f_{3}$. The feature labeled inclined interface [Fig. 3(b)] occurs at the beat frequency $f_{L}-f_{3}$. The starting point for the inclined interface is near the interface zone. The inclined interface extends spanwise across the modulated zone on a significant angle.

The presence of the inclined interfaces leads one to suspect that the actual spanwise length of the modulated zone might be changing in time. A conventional power spectrum yields a strongly quasiperiodic spectrum at specific spanwise locations. The local frequency analysis shows that there is a variation of the shedding frequency in time within the modulated region.

\section{Indirect mode at low Reynolds number}

The simplest occurrence of the indirect mode was at very low Reynolds numbers, when there was no shedding behind the small cylinder $\left(f_{S}=0\right)$. The interface zone was periodic (at the modulated region frequency $f_{3}$ ), and the large-wake shedding frequency was $f_{L}$. Inclined interfaces occurred at the beat frequency $\left(f_{L}-f_{3}\right)$. Flow visualization is seen in Figs. 4(a) and 4(b) for $\operatorname{Re}_{L} / \operatorname{Re}_{S}=67 / 38$. Two photographs show the complete cycle. Figure 4(a) shows an inclined interface. The inclined interfaces occur with very low frequency, so they are not always seen [Fig. 4(b) ]. We determined from a two-probe study of the wake that the process of formation of the inclined interfaces was periodic.

The variation of local frequency at various spanwise positions is shown in Fig. 5. The minima in the data sets of Fig. 5 were aligned to accentuate the periodicity. The $36 d_{L}$ position is pure-wake shedding, showing the variation in frequency that is associated with normal-wake shedding. This is the background level. Normal shedding also occurs in the interface zone, at $2.75 d_{L}$. The local frequency in the modulated zone (from $\sim 3$ to $11 d_{I_{l}}$ ) varies periodically in time at the beat frequency $f_{L}-f_{3}$.

The amplitude of the frequency variation increases with distance from the interface, reaching a maximum at $9.2 d_{L}$. It then decreases rapidly to background level at $11 d_{L}$. The most quasiperiodic traditional spectra were seen at the $9.2 d_{I}$.

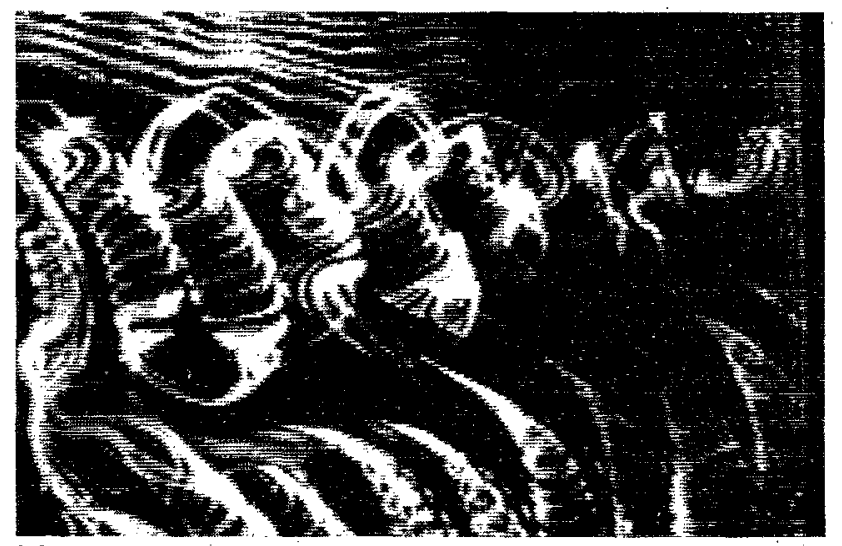

(a)

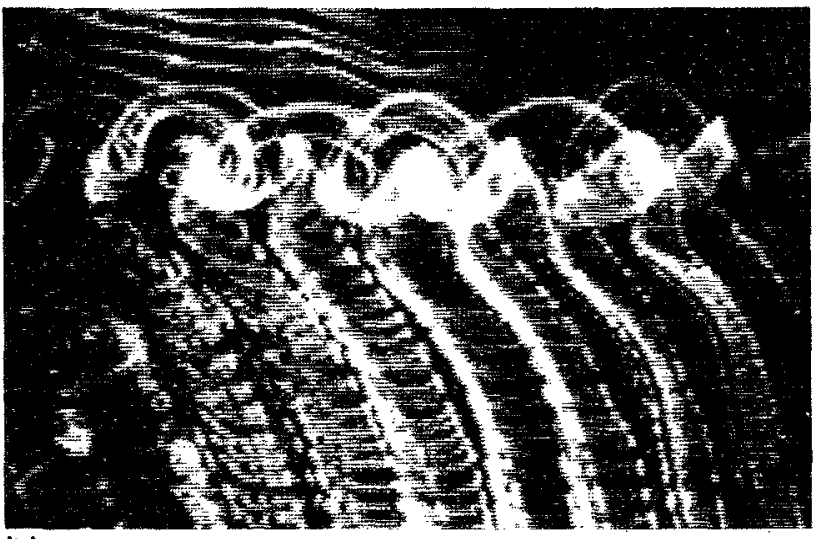

(b)

FIG. 4. (a) The inclined interface region of the Reynolds number $67 / 38$ case for the 1.76 diameter ratio. (b) The appearance of the flow when the inclined interfaces are not present. 


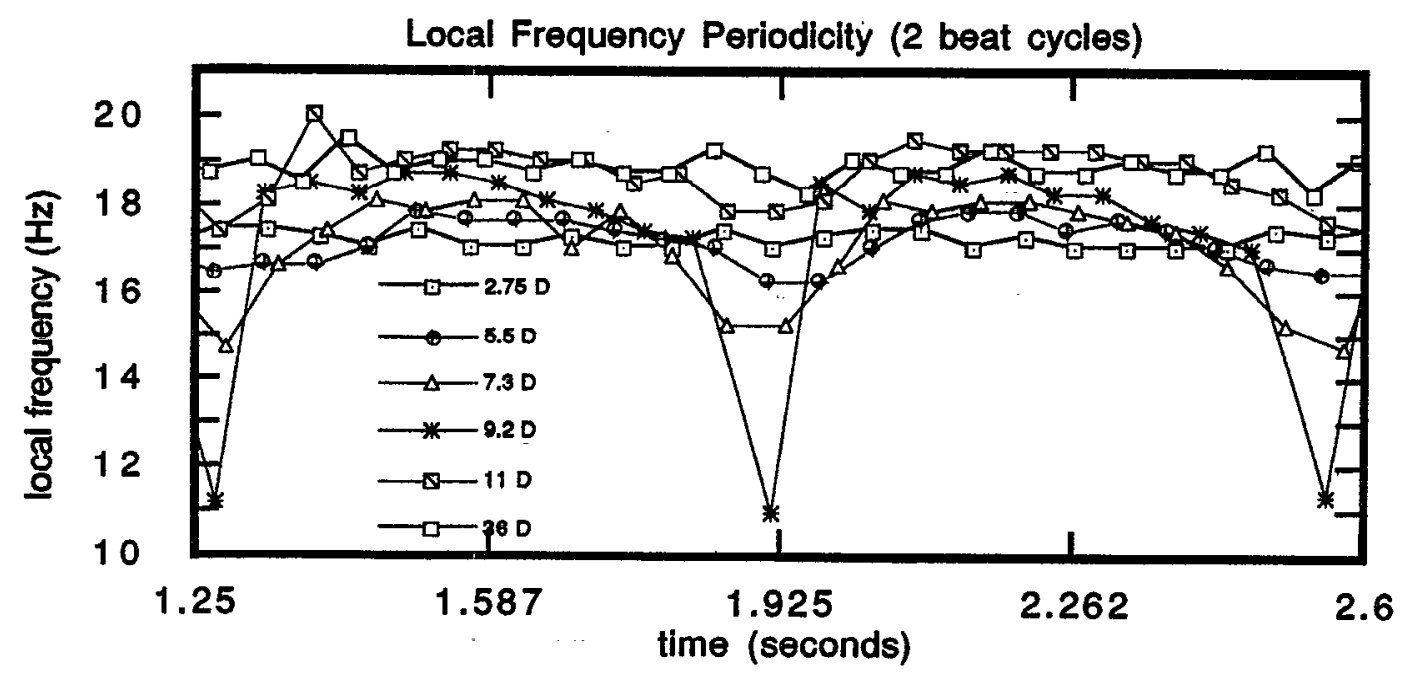

FIG. 5. Variation of local frequency with spanwise position (given in terms of $D$, large diameters) for the modulation region of the indirect mode. Here, $\operatorname{Re}_{L} / \operatorname{Re}_{S}=67 / 38$.

position. Figure 6 shows mean frequency and maximum frequency variation (as determined by three times the standard deviation) as a function of spanwise position in the modulation region. The mean local frequency makes a substantial jump at the $9.2 d_{L}$ position.

The relative phase of the vortex structures can be compared from the results of the local frequency analysis for the two-probe measurements. A stationary probe was placed in the interface zone, at $2.25 d_{L}$, while the other probe sampled along the span. (The downstream position was $10 d_{L}$ and the probes were $2 d_{L}$ from the wake center.) Four sets of simultaneous phase measurements are presented in Fig. 7 for different locations along the span. Points of equal phase are represented by vertical lines. The grid lines correspond to the period of the beat frequency $(0.64 \mathrm{sec})$.

The cnd conditions of the vortices at the interface zone cause the $2.25 d_{L}$ vortices to be nearly $180^{\circ}$ out of phase with the vortices of the modulated region at $5.5 d_{L}$. The vortices in the modulation region are 1:1 in number, all the way out to $9.2 d_{L}$. The periodic phase oscillation occurs as a result of the variation of local frequency. At the $9.2 d_{L}$ position, there is a

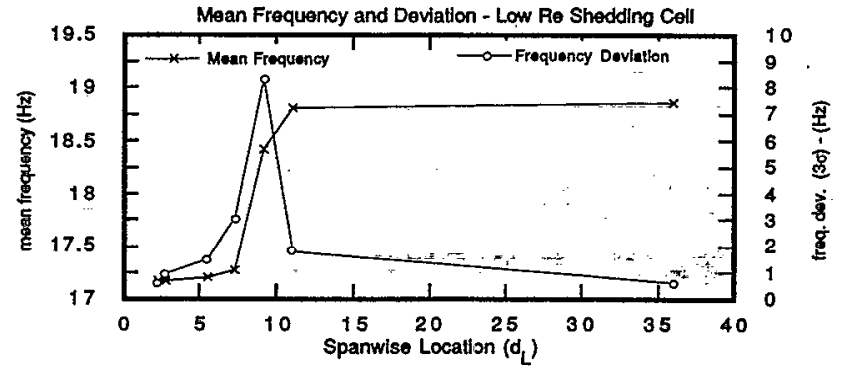

FIG. 6. Mean local frequency and deviation versus spanwise position on the large-diameter side for the $\mathrm{Re}_{L} / \mathrm{Re}_{S}=67 / 38$ case for the indirect mode. gap that corresponds to the low local frequencies found here. This low frequency is a subharmonic of the shedding frequency in the cell, indicating the loss of one structure per beat. This is most easily seen by counting the vortices in the $2.25,11 d_{L}$ set over one beat cycle. There are 11 vortices formed in the modulated zone for every 12 formed in the wake.

In summary then, even though there is local frequency variation within the cell region that affects the mean frequency determined by a local analysis, the same number of vortices is being formed throughout the modulated region. There is a narrow zone in which the number of vortices being formed changes, and for this case, there was simply the loss of $\sim 1$ vortex structure per beat on each side of the larger diameter vortex street. The frequency of vortex formation was locked to a ratio of $12 / 11$.

Since the location at which the number of vortices being

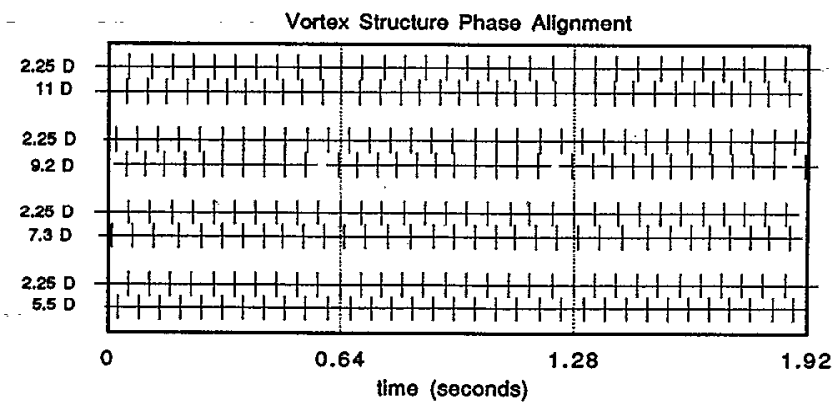

FIG. 7. Phase relationship hetween reference probe near the interface, and various spanwise positions (given in terms of $D$, large diameters) within the modulation region of the indirect mode. Vortex structures are $1: 1$ until the $9.2 d_{L}$ position. Farther out, there is a loss of $\sim 1$ vortex structurc per beat cycle. 
formed is fixed, the appearance of the inclined interfaces must be due to small gaps in the vortex pattern. The twoprobe local frequency analysis allowed us to check this. The gaps are identified by the low local frequencies of the modulated region. In all our data, the lowest local frequencies appeared at the minima of the amplitude modulation envelope. Periodic variation in the amplitude of the wavelet coefficient can be found throughout the modulation region, even where the local frequency variation is negligible. We used conditional sampling based on the wavelet coefficient values to find the relative time positions of the low frequencies. The result of this analysis showed that there was a time lag that increased with spanwise distance from the interface. Figure 8 shows the time of the minimum wavelet coefficient (the time of the low-frequency events) versus spanwise location for three beat cycles of data (one beat per plot). The grid lines give the approximate spacing of the vortex lines, not the actual positions of the vortices. (The ratio of vortex wavelength to cylinder diameter was assumed to be 4.) The time axis runs from right to left, to allow comparison with the flow visualization (Fig. 4).

Comparison of these two figures shows that the position
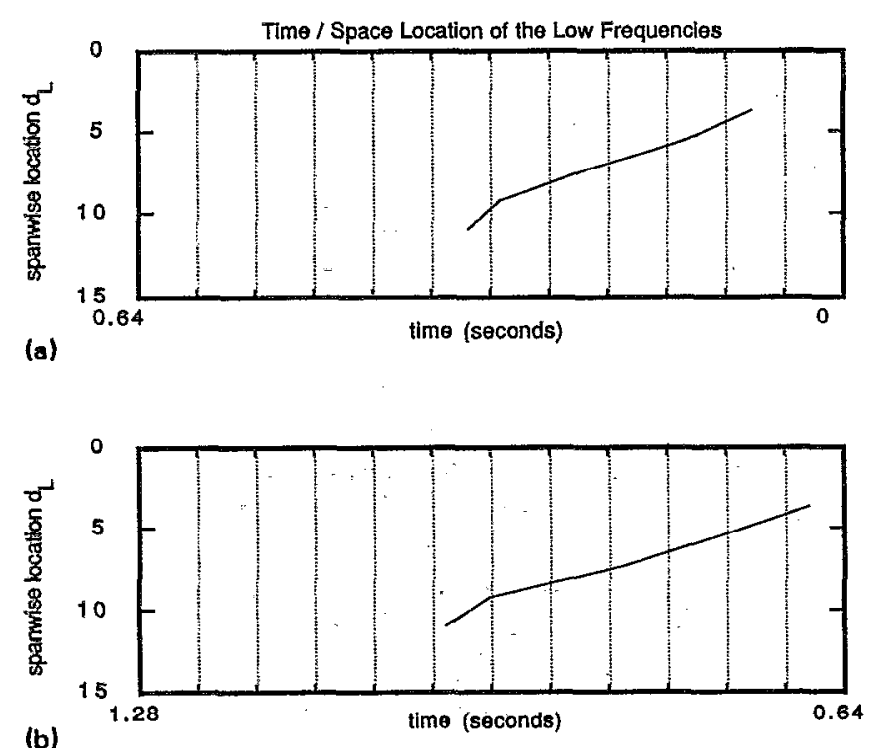

(b)

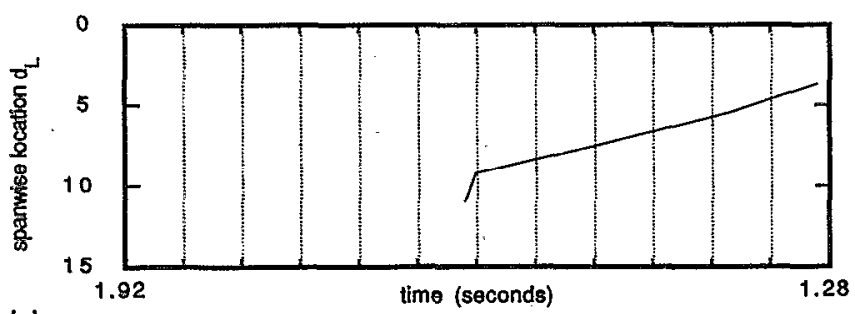

(c)

FIG. 8. Spanwise position of the low-frequency events that cause the inclined interfaces in the modulation region of the indirect mode. The position at which the frequency of vortex formation changes is at the kink or elbow of the interfaces shown. The grid lines give an approximate mean vortex spacing in the shedding cell region, not the actual vortex positions. of the low frequencies (the gap position) coincides with the inclined interfaces. The spacing variation produced by the frequency modulation process causes the inclined interfaces in the modulated region of the wake. The gap position changes with time, moving away from the interface. Initial$1 y$, the gap distance is very small, but it grows in time while as it moves in space. Growth of the waveform modulation envelope is coupled to the growth of the gap (the decrease in local frequency).

\section{B. Interaction region behavior as a function of Reynoids number and diameter ratio}

\section{1. $1.55<r$}

For each of the three diameter ratio categories, we studied the interface behavior as a function of the Reynolds number within the laminar shedding range. In addition, we studied in detail the $\mathrm{Re}_{L}=99$ case, taking spanwise measurements at the wake center (the position of minimum velocity in the wake), at downstream positions of 2 and $15 d_{L}$. Spectral, velocity, and local frequency data were taken as a function of the spanwise position for each of these cases.

The $1.76 r$ case was studied as representative of this highratio category. Only the indirect mode of wake interaction was observed for this diameter ratio, for all Reynolds numbers considered. The low Reynolds number case in which the small diameter wake was not shedding was discussed in Sec. III A 2 as a simple example of the indirect mode. When the small diameter wake begins shedding, the dynamics of the interface region becomes more complicated and the modulation region dynamics is slightly more complex.

The $\operatorname{Re}_{L}=99$ case shows these complications, as well as the effect of increasing Reynolds number. Figure 3(a) shows flow visualization for this case. When the small-diameter side was not shedding, there was little occurring in the interface region except for the vortex end behavior of the finite length street. Once shedding begins behind the smalldiameter cylinder, the vortex linkages of the interface region must accommodate the neighboring vortex street. A traditional spectrum and the corresponding local frequency behavior for data taken in the interface region $10 d_{L}$ downstream of the diameter discontinuity is shown in Figs. 9(a) and 9 (b), respectively. Peaks in the spectrum are at $10.6 \mathrm{~Hz}$, $18.9 \mathrm{~Hz}$ (beat frequency), $29.5 \mathrm{~Hz}$ (cell shedding frequency), $48.6 \mathrm{~Hz}$ (small-diameter wake shedding frequency), and others. The local frequencies at the interface $(O D)$ can be seen to correspond closely to subharmonics of the shedding frequencies. The $18.9 \mathrm{~Hz}$ beat period corresponds to the time between each of the local frequencies (the variation in this is reflected by the spread of the peak). Last, the $10.6 \mathrm{~Hz}$ frequency, which is not quite a subharmonic of the beat frequency, can be seen to be caused by the variation between the two local frequencies, a somewhat periodic variation that is more regular than the beat period.

Figure 10(a) shows the spanwise distribution of frequency components from conventional FFT's for this case. The frequency resolution is $0.24 \mathrm{~Hz}$ and the downstream position is 2 diameters. The spanwise velocity profiles were 

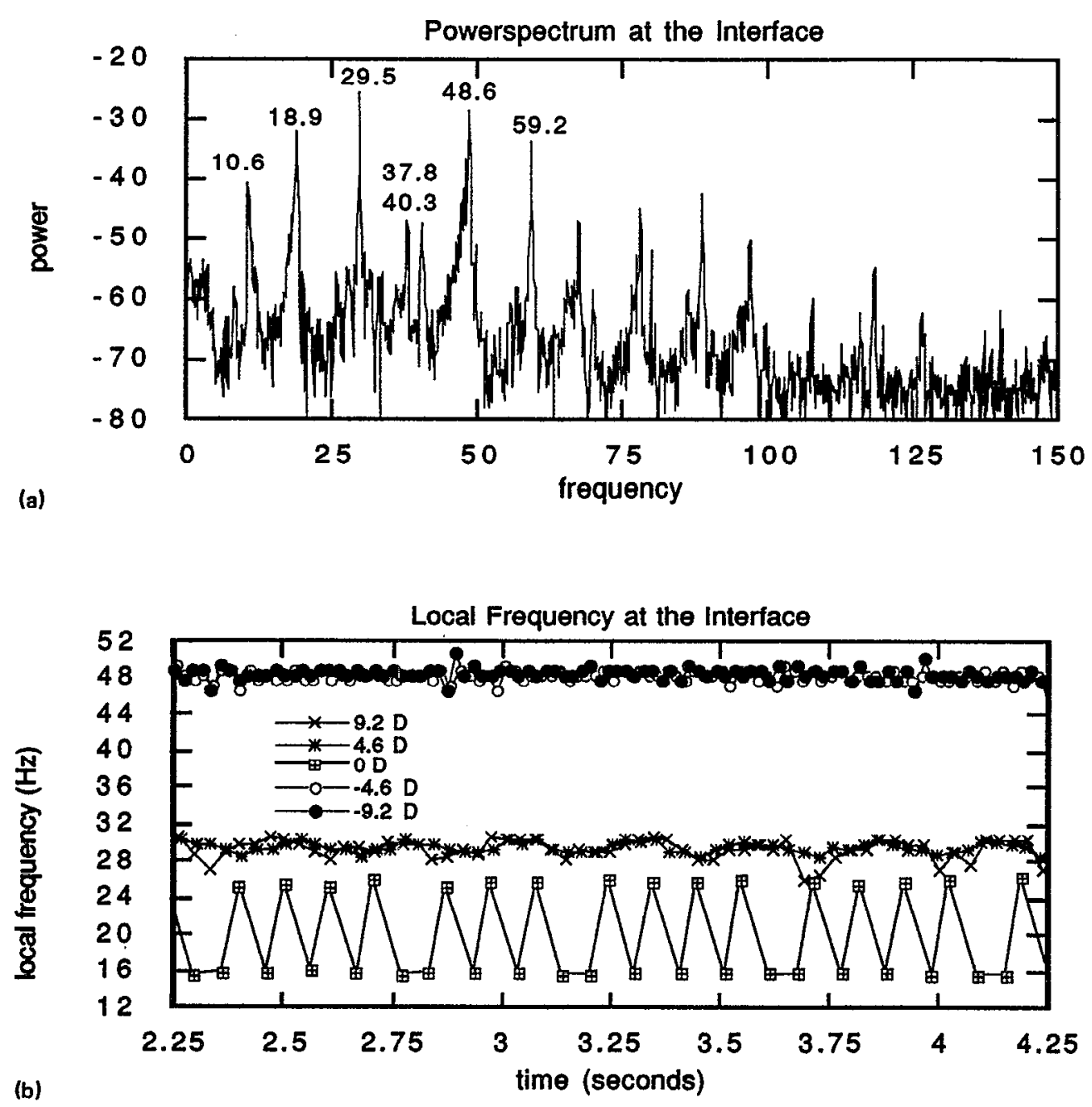

FIG. 9. (a) The power spectrum for the $\operatorname{Re}_{L}=99$ case for the indirect mode at the interface $(O D)$. (b) The local frequency analysis of the same data, at various spanwise positions, given in terms of $D$ (large diameters) from the interface (Negative positions are in the small diameter wake.) taken at the wake center. Figure 10(b) is the mean defect velocity and 10 (c) gives the broadband rms or fluctuation velocity. Regions I and II are stable wake shedding zones. The modulation region spans 25 diameters. This is twice the span of the low Re number case presented in Sec. III A 2. The lines marked $A$ and $B$ give the spanwise locations of the peaks in the fluctuation velocity profiles at the $15 d_{L}$ downstream [10(c)], and are included as convenient reference points. These peaks in the fluctuation profiles correspond to amplitude minima in shedding frequency and maxima in beat frequency. The peak at $A$ is due to the vortex interaction caused by the discontinuity itself, while the peak at $B$ is a consequence of the modulation region dynamics. There is a noticeable peak in the very near wake mean velocity profile, which occurs at $13.8 d_{L}$, labeled $B$ in the figures.

The point at $B$ appears to be significant, and the local frequency analysis shown in Fig. 11 (a) helps to explain why this is so. Point $B$, at $13.8 d_{L}$, is the farthest point at which the low-frequency variations shown in Fig. 11 (a) occur regularly. However, there is significant variation present in the modulation region over long time spans, as can be seen in Fig. 11(b). Figure 11(a) can be compared to Fig. 5 from
Sec. III A 2. There is similarity in the short-term local periodicity of Fig. 10 (a), in the 0 to $13.8 d_{L}$ range. The long term variation of this higher Reynolds number case was not present in the lower Reynolds number case. This was also noticeable in flow visualization, in which the regularity of the inclined interfaces was more noticeable at the lower Reynolds numbers. Another noteworthy difference between high and low Reynolds number behaviors is that, while the spanwise length of the modulation region increases with Reynolds number, the point at which there is an actual change in the frequency of vortex formation has not changed significantly (from $9.3 d_{L}$ ), but has spread out laterally (from 9.2 to $\left.13.8 d_{L}\right)$. The lateral spreading causes the variation shown in Fig. 11(b).

The $13.8 d_{L}$ position is the farthest spanwise position in which a truly quasiperiodic spectrum can be found [Fig. 12(a) ]. Past this point, there is spectral broadening of the peaks, as seen at $14.9 d_{L}$ in Fig. 12(b). The spectral broadening of the peaks decreases with spanwise position, and the spectrum of a normally shedding laminar street is again found at $25 d_{L}$. [This can be seen in Figs. $10(\mathrm{~b})$ and $10(\mathrm{c})$, in which $25 d_{L}$ is the position at which the mean defect velocity 

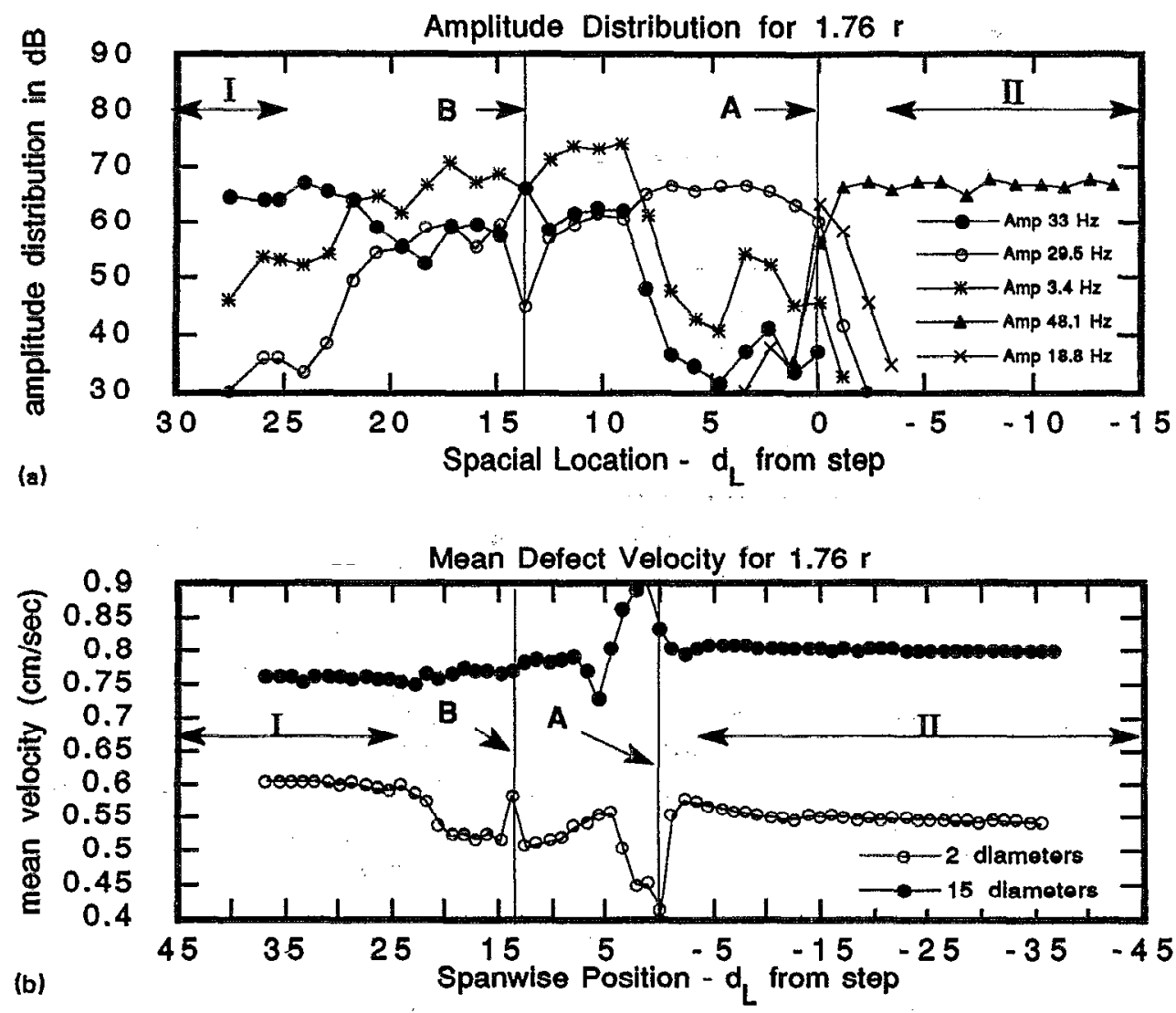

FIG. 10. Results from the spanwise measurements taken at the wake center for the $\operatorname{Re}_{L}=99$ case of for the indirect mode. (Negative positions are in the small diameter wake.)

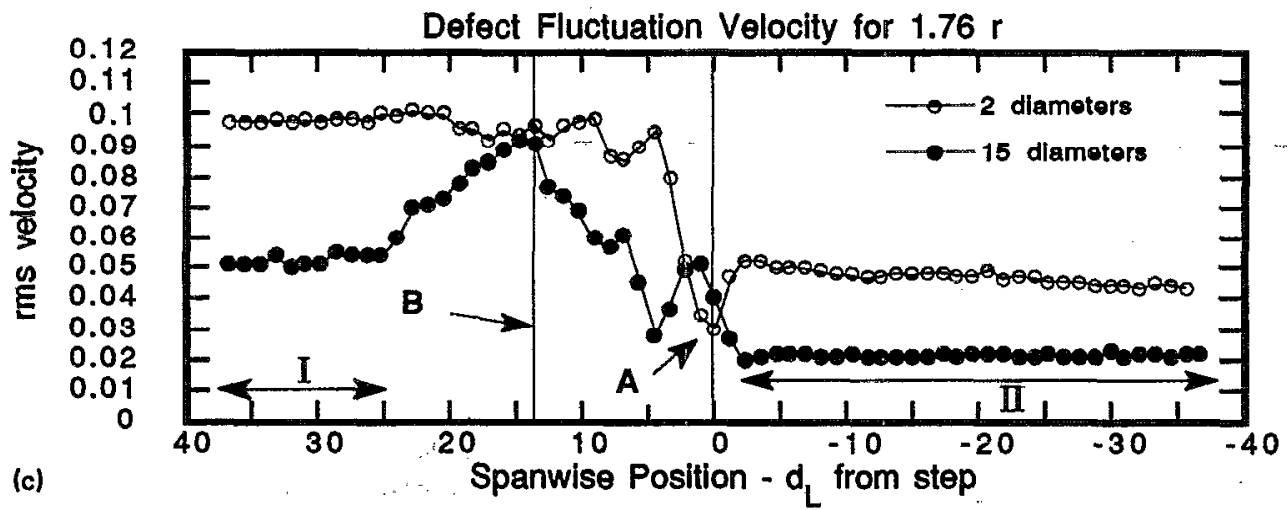

stops changing.],

Figure 13 shows the mean frequency and maximum frequency deviation behavior versus span position. The dynamics at the diameter discontinuity can be seen to be rather distinct from the modulation region dynamics. The frequency deviation becomes negligible at the 5 diameter position before increasing again in the 9 to $13.8 d_{L}$ span. The increase in variation that occurs for this case may be due to the increase in length of the modulation zone or the increase in Reynolds number. (Differences between Figs. 6 and 13 aid in showing the increased complexity of the modulation region dynamics for higher Reynolds numbers.)

\section{2. $1.25<r<1.55$}

For this range of diameter ratios, the mode of interaction appeared to be a function of Reynolds number. Within this range, diameter ratios of $1.27,1.34$, and 1.53 were studied.

For these ratios, the appearance of the direct mode coincided with the appearance of angled shedding throughout the large-diameter wake. The shedding angles associated with the direct mode seemed unusually steep, so the shedding angle in the $d_{L}$ and $d_{S}$ wakes was systematically studied for 1.34 and 1.53 diameter ratios. Baseline data for the angle of shedding of plain two-dimensional cylinders were ob- 


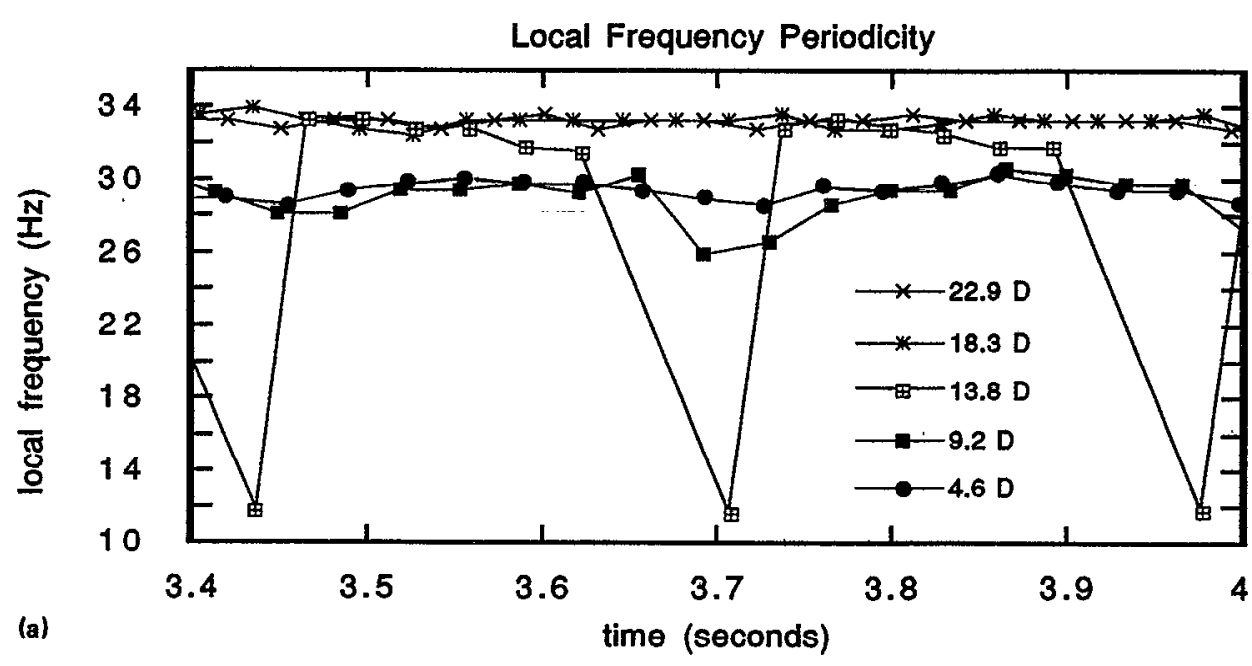

Local Frequency Variation in Time

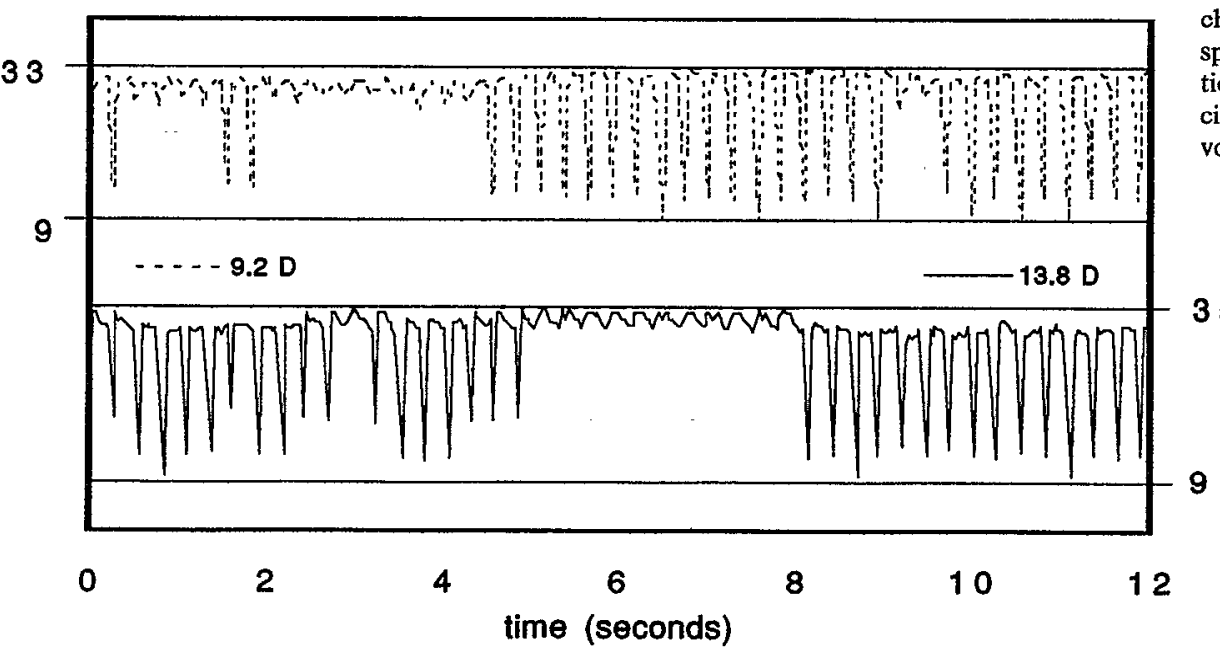

FIG. 11. (a) Variation of local frequency with spanwise position (given in terms of $D$, large diameters) for the modulation region of the $\operatorname{Re}_{L}=99$ indirect mode. (b) Long-term local frequency behavior for this data. This shows that the position at which the frequency of vortex formation changes varies within the 9.2 and 13.8 spanwise position. It does not change position very quickly; each low-frequency oscillation represents the formation of $\sim 10$ vortex structures. tained (for 0.157 and $0.211 \mathrm{~cm}$ cylinders, with $A R=387$ and 288 , respectively) for comparison purposes, and is shown in Fig. 14(a). All cylinders ended at wall boundary layers. The plain cylinders display very specific trends, but have substantial error bars, which (measurement details in Sec. II A)) represent temporal fluctuations in angle for $\operatorname{Re}>70$. The measured angles of shedding for the stepped cylinders are shown in Fig. 14(b). Aspect ratios for the 1.34r case were $A r_{L}=145$ and $A r_{S}=194$, and for the $1.53 r$ case were $A r_{L}=126$ and $A r_{S}=194$. (See Fig. 1 and Table I.) The shedding angle in the small-diameter wake did not change appreciably from the baseline measurements. In contrast, shedding angles in the large-diameter wake increased substantially, and were nearly constant in the ranges $70<\operatorname{Re}<130$ ( 1.34 case) and $78<\operatorname{Re}<134$ ( 1.53 case).

Temporal variation was negligible in these ranges. The shedding angle for the $1.53 r$ case was larger than for the $1.34 r$ case. The sign of the angle is included because the direction of the shedding angle in the direct mode was always in the positive direction (shown in Fig. 2).
The direct mode of interaction occurrcd for the range of Reynolds numbers for which the $d_{L}$ wake shedding angle was steep and fixed [the plateaued section of oblique shedding angle in Fig. 14(b) ]. The indirect mode occurred for all laminar Reynolds numbers studied below and above these ranges.

The $1.34 r$ case was studied for $\operatorname{Re}_{L}=99$, to match Secs. III $B 1$ and 3. A power spectrum in the interface region [same position as in Fig. 9(a)] is shown in Fig. 15(a). Base shedding peaks are 69.5 and $55.1 \mathrm{~Hz}\left(f_{S}\right.$ and $f_{L}$, respectively). Other peaks in the spectrum are caused by nonlinear interaction (sums and differences) of the base shedding peaks.

Figure 15(b) shows a portion of the local frequency data, at different spanwise locations. At the interface $(0 D)$, the local frequencies observed were always either $\sim 60$, $\sim 25$, or $\sim 18 \mathrm{~Hz}$. Figure 16 is a schematic that shows our interpretation of the local frequency data. The vortices that connect across the interface have a spacing that is intermediate to the vortex spacing of the neighboring streets, causing 

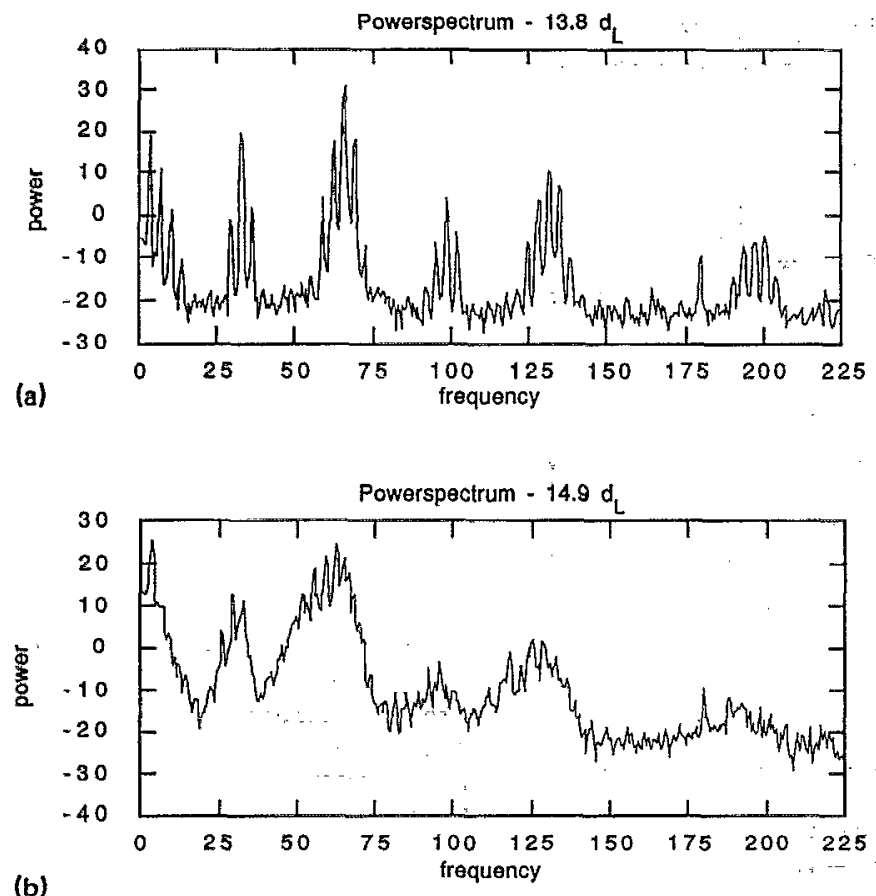

FIG. 12. (a) Power spectrum for the $\operatorname{Re}_{L}=99$ indirect mode $13.8 d_{L}$ from the diameter discontinuity on the large-diameter side. (b) Power spectrum for the $\mathrm{Re}_{L}=99$ indirect mode $14.9 d_{L}$ from the diameter discontinuity on the large-diameter side. A sudden loss of quasiperiodicity is seen just beyond the farthest location of the inclined interface region.

the $60 \mathrm{~Hz}$ value of local frequency (intermediate to $f_{S}$ and $f_{L}$ ). The low local frequencies $(25$ and $18 \mathrm{~Hz}$ ) are very nearly the 3 rd and 4 th subharmonics of $f_{S}$, and the 2 nd and 3 rd subharmonics of $f_{L}$. These frequencies represent gaps in the vortex connections across the interface. These gaps cause the hole region (discussed in Sec. III A 1) that is bordered by sequences of vortex half-loop linkages (Figs. 2 and 16).

The schematic shows why the low local frequencies might jump from 18 to $25 \mathrm{~Hz}$ : A probe located to the white vortex side of the wake center of Fig. 16 would yield $18 \mathrm{IIz}$, while a probe on the black vortex side of wake center would yield $25 \mathrm{~Hz}$. There are two or three strong vortex connec-

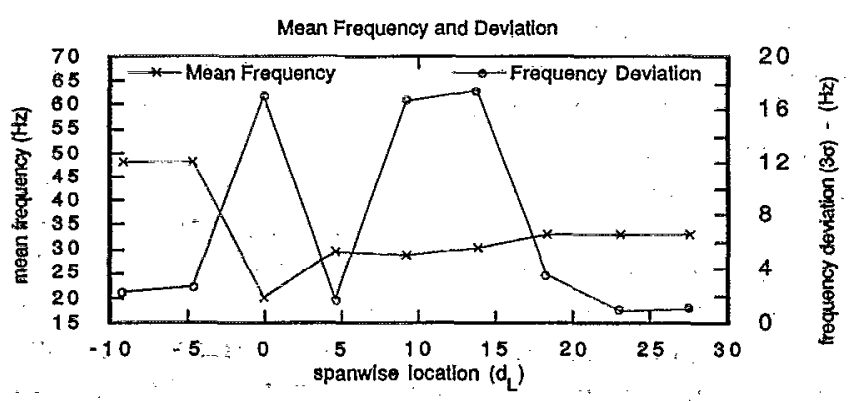

FIG. 13. Mean local frequency and deviation versus spariwise position on the large-diameter side for the $\mathrm{Re}_{L}=99$ case for the indirect mode. (Negative positions are in the small-diameter wake.) tions across the interface in any one beat cycle, on each side of the vortex street. The variation of the low local frequency may result from the method, the physics, or both. The strength of the vortex linkages at the edge of the hole may be very close to the cutoff value, so that this vortex is included only half of the time. This would cause a variation from 18 $25 \mathrm{~Hz}$. On the other hand, if there were five strong vortices of both signs for every beat cycle, the data readings would be unpredictable since the probe is located on one side of the wake. Only two or three strong linkages would be seen in the data for each beat cycle, and this could also cause the variation. In any case, the intent of Fig. 16 is to represent the types of vortex linkages that are suggested by our studies, not the exact pattern.

The vortex linkage pattern is a simple matter of geometry, with the linkage forming to the nearest available neighbor, and the excess vorticity going to the next available site. The actual ratio of vortex shedding frequencies for this case was $1.27 \mathrm{~Hz}$. There are $\sim 5 / 4$ vortices of each sign; involved in one beat cycle.

Flow visualization for the $\operatorname{Re}_{L}=99$ case is shown in Fig. 17. The $d_{L}$ wake is shedding obliquely. Spanwise measurements for this case are given in Fig. 18. The spanwise distribution of frequency components is shown in Fig. 18(a). Figure 18(b) gives the mean defect profile and 18(c) is the fluctuation profile. A comparison of Figs. 18 and 10 shows that the features that characterized the modulation region are absent from Fig. 18. There is no third frequency and the region of frequency interaction is confined to a narrow spanwise region. The region of lower mean defect velocity is gone, as well as the high-fiuctuation region marked B in Fig. 10. When the interaction is in the direct mode, stable vortex streets form within 5 diameters of the discontinuity.

\section{3. $1.25>r$}

Only the direct mode of interaction occurs in this category, which is very similar to all the direct interaction cases discussed so far. The $1.14 r$ case was used as representative of this category. However, the effect of the discontinuity was not strong enough to control substantial regions of the span, as it was for 1.27 ratios and larger. Therefore oblique shedding occurs but with some temporal variations in shedding angle. This variation in shedding angles may be caused by the wall boundary layers rather than by the discontinuity.

\section{DISCUSSION AND CONCLUSION}

\section{A. Modes}

The wake of a cylinder with a discontinuity in diameter showed two distinct modes of behavior that were a function of diameter ratio and Reynolds number. These were called the direct and indirect modes of interaction. The direct mode describes a frequency (vortex) interaction that takes place in a very narrow region at the interface between the higher and lower Reynolds number wakes. The presence of a plateau in a shedding angle versus Reynolds number curve for the large diameter wake is an identifying characteristic of the direct mode. The indirect mode describes a frequency interaction that includes a frequency-modulated region in the 

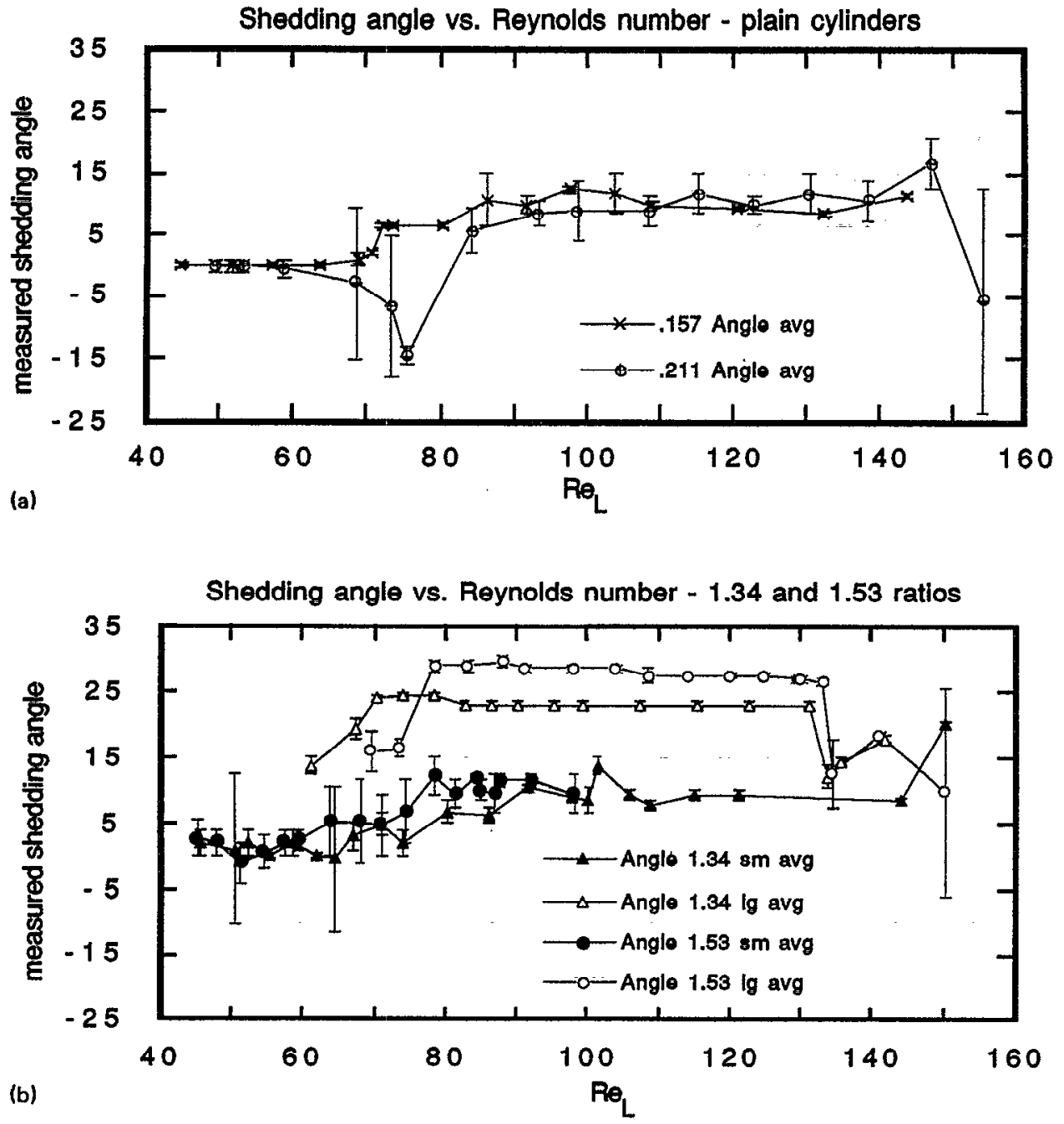

FIG. 14. (a) Baseline angle of shedding measurements for our facility. (b) Angle of shedding measurements for the 1.34 and $1.53 r$ cases. The direct mode of interaction occurred along the plateaued region of shedding angle. wakc of the larger diameter cylinder. For the indirect mode, there are three shedding frequencies involved, and two locations at which vortex interactions and linkages occur. A crude estimate of the border between the two modes is given in Fig. 19.

There would appear to be a complex set of parameters that determines the odd borders between the direct and indirect modes. We can compare the inclined interface region observed in our investigation to similar features seen in other flows. Within the inclined interface region, there is a substantial phase shift (or phase dislocation) between neighboring vortex lines. This phenomenon has been seen to occur in wakes, for example, in Williamson, ${ }^{8}$ Figs. 6 and 23 therein. These phase dislocations are caused by the cylinder endplate shedding cells.

Spontaneous and induced phase dislocations have been observed in mixing layers. Figure 17 of Browand and $\mathrm{Ho}^{9}$ shows an inclined region of phase change in the mixing layer, which is similar in appearance to our inclined interfaces. Huerre $^{12}$ suggests that a secondary instability initiated by a pattern inhomogeneity can induce a localized vertical vorticity field that might result in such a flow feature. Although the two-dimensional mixing layer is the subject of Huerre's analysis, the derivation can be generalized to other shear layers. There are some similarities in the behaviors of the wake and mixing layer three dimensionalities. We will not compare these two flows directly, but will discuss the inclined interfaces as a form of secondary instability.

We will first discuss formation of the inclined interfaces in the larger-diameter ratio (1.76) case. The inclined interfaces are regions in which the amplitude and local frequency of the velocity fluctuations are reduced. This reduction increases with spanwise distance from the interface, and the amplitude goes to zero in a narrow region in which the vortices appear pinched off. We believe this process may be caused by the growth of an instability initiated by the mean shear caused by the difference in the mean velocity profiles of the two wakes. The mean shear creates a local vertical vorticity field that initiates an anisotropic disturbance that propagates in the spanwise (and perhaps streamwise) direction. As the instability grows, it appears to locally destroy or modulate the two-dimensional vortex shedding.

The indirect mode initiates the secondary instability periodically at the beat frequency between the large wake shed- 


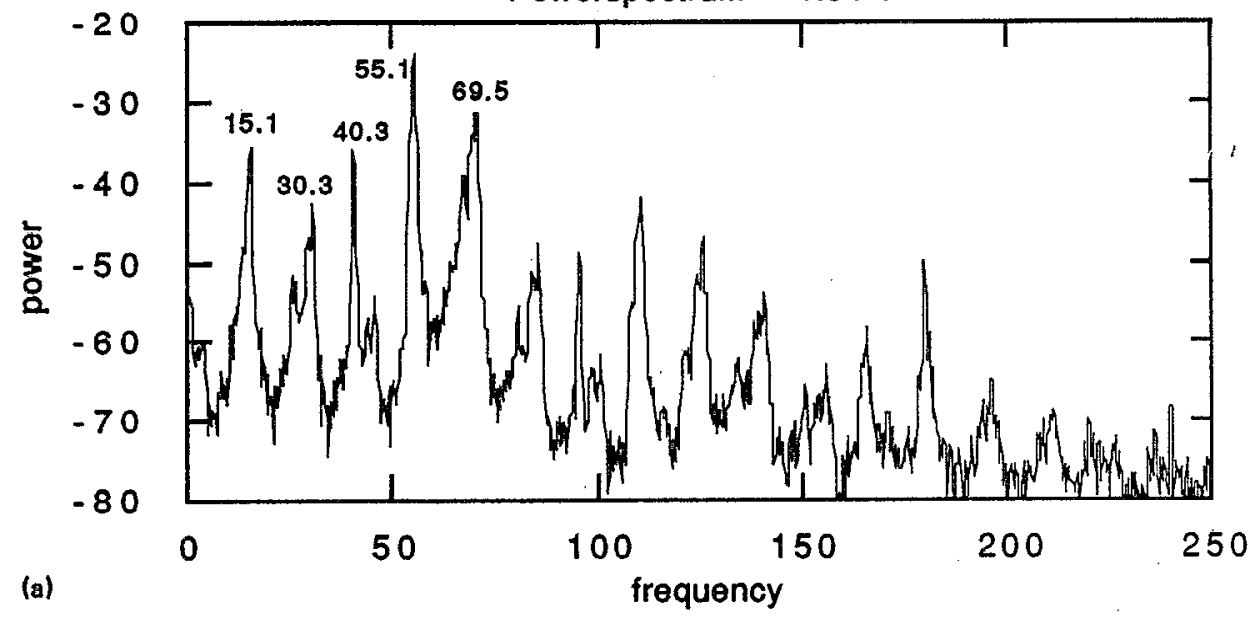

(b)

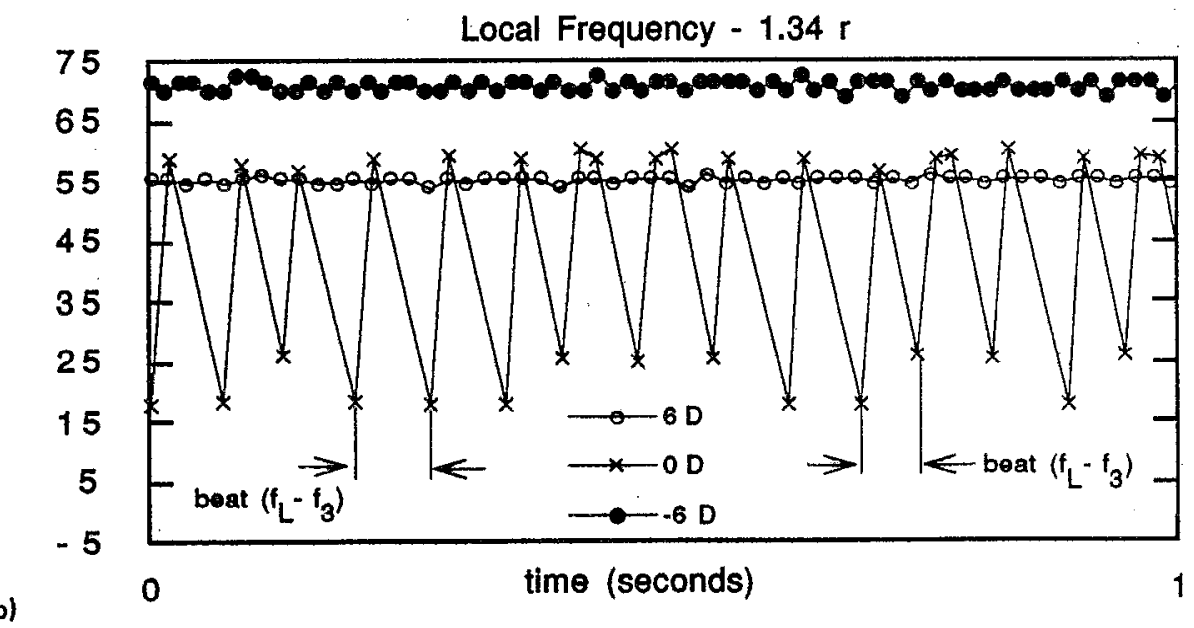

FIG. 15. (a) The power spectrum for the $\mathrm{Re}_{L}=99$ case for the direct mode at the diameter discontinuity. (b) The local frequency analysis of the same data, at various spanwise positions, given in terms of $D$ (large diameters) from the interface. ding frequency and the lower shedding frequency of the modulation region. (We do not know how this is accomplished.) The number of interfaces appearing along the span at any one time is a function of the spanwise speed of propagation, the growth rate, and the frequency of initiation of the secondary instability. Since we observed no simple relationship between these variables and Reynolds number, we did

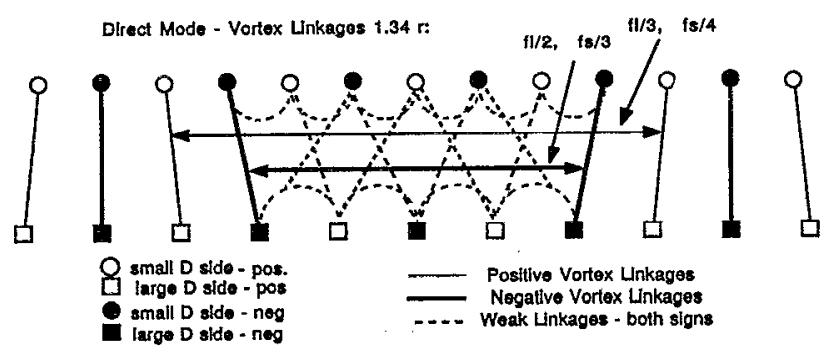

FIG. 16. Schematic showing the types of vortex linkages for direct mode $\left(\mathrm{Re}_{L}=99\right)$. This is a view of the interaction region (see Fig. 1). The circles represent the ends of the vortices of the small-diameter wake and the squares represent the ends of the vortices of the large-diameter wake. Positively signed vortices are white and the negative vortices are black. not pursue this aspect of the problem. At very low Reynolds numbers, there was never more than one interface observed along the span at any one time (Fig. 4). However, in general, the frequency of initiation appeared to increase rapidly with Reynolds number, so more than one inclined interface formed along the span at any one time at higher Reynolds numbers.

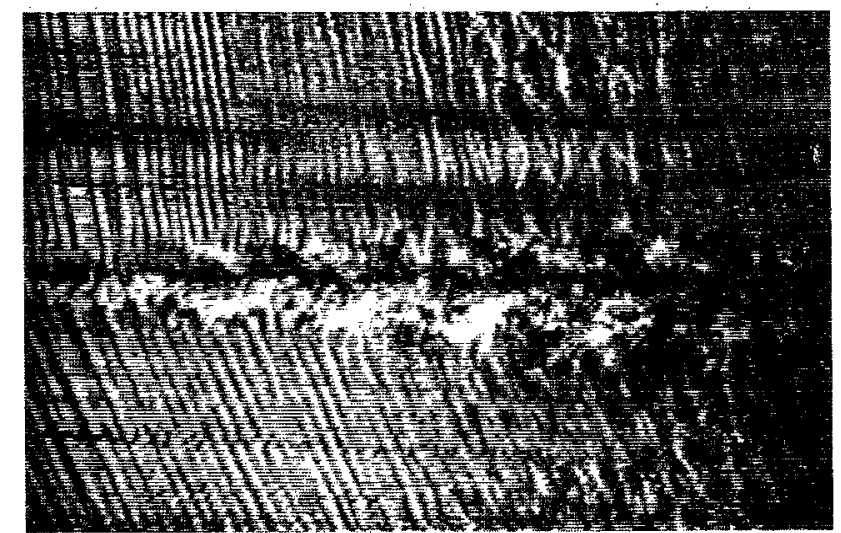

FIG. 17. Flow visualization for the 1.34 diameter ratio at a Reynolds number of $99 / 74$. This is the direct mode. 


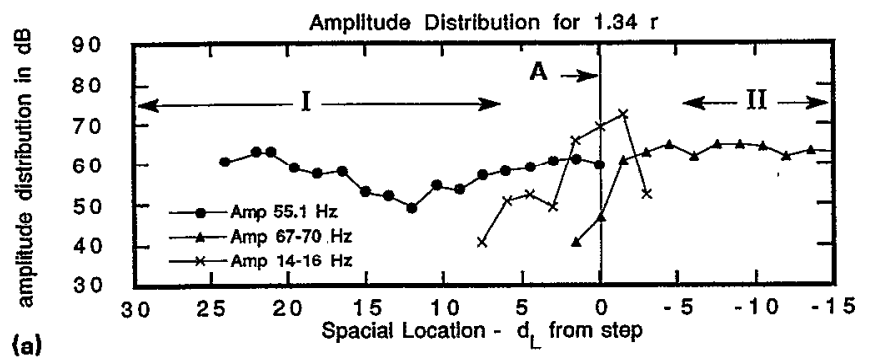

(a)
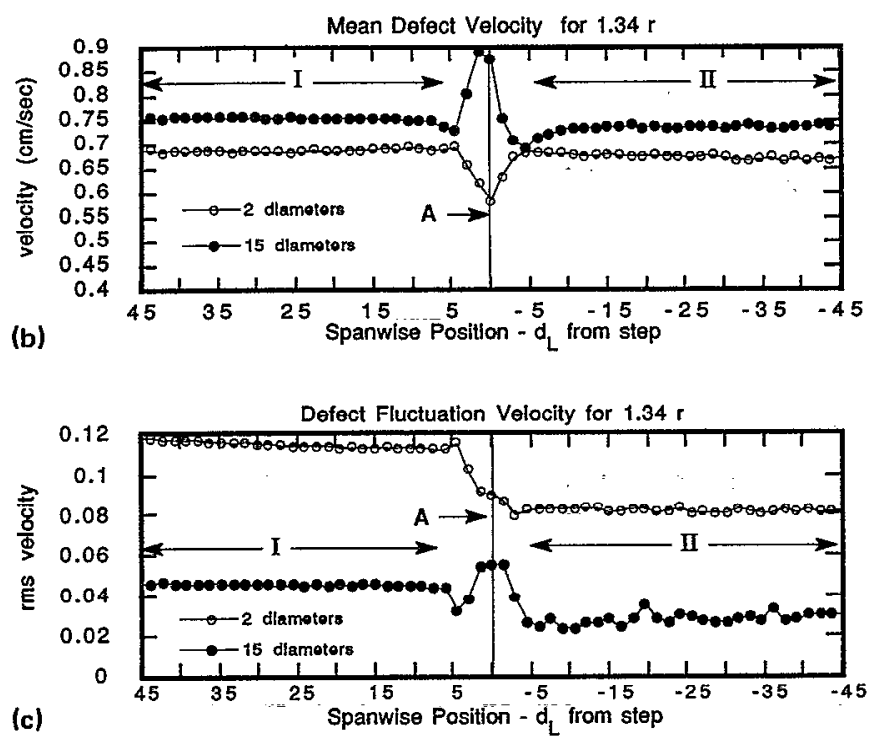

FIG. 18. Results from the spanwise measurements taken at the wake center for the $\operatorname{Re}_{L}=99$ case of for the direct mode. (Negative positions are in the small-diameter wake.)

The moderate diameter ratio modes fall into three distinct ranges (Fig. 19). The behavior of the disturbances found in regions 1 and 2 are very similar to the higher-diameter ratio case. In region 1 , the disturbances can be clearly seen, but the repetition rate is very low. The disturbances are not as apparent in the near wake, but become more noticeable with downstream distance. The spanwise position of the

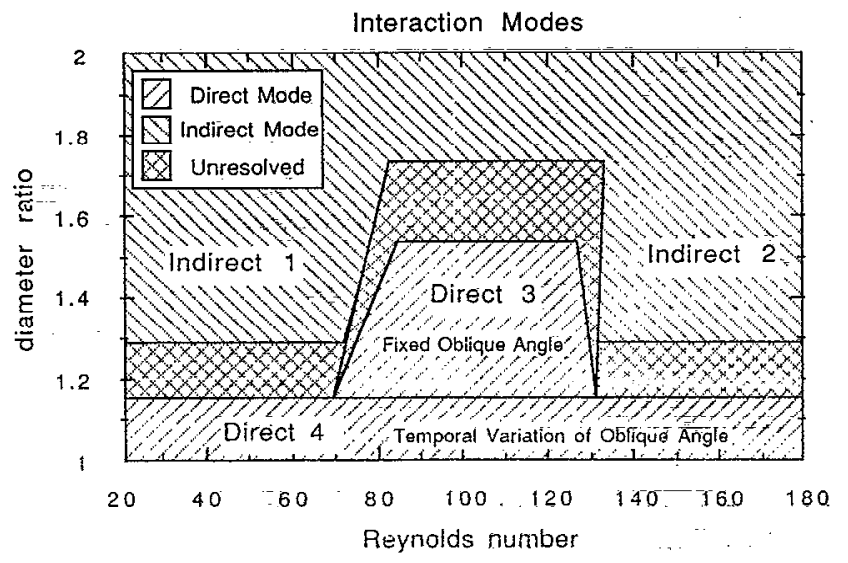

FIG. 19. Mode appearance as a function of Reynolds number and diameter ratio. maximum disturbance amplitude (the farthest spanwise position of the inclined interfaces) moves outward from the interface. Probe measurements extended $40 d_{L}$ from the discontinuity. The inclined interfaces were found at the $40 d_{L}$ position at a Reynolds number of 70 (the border of regions 1 and 3). The modulation region is very wide, and the vortices can be seen to shed obliquely in the region between the inclined interface and the discontinuity. The growth rate of the moderate ratio disturbances was lower, and amplitude modulations were negligible near the discontinuity These observations suggest that the direct mode may be a Reynolds number/diameter ratio range over which the disturbances propagate along the whole span of the cylinder before the amplitude growth is noticeable. So, the direct mode may be caused by the extension of the indirect mode over the entire span of the cylinder when it has a slow rate of growth.

\section{B. Vortex linkages}

For low- and moderate-diameter rátios, the vortex linkages at the diameter discontinuity exhibited very specific behaviors. A schematic of one case was included as Fig. 16, which is representative of the behavior of the vortices at the interface for the direct mode. Differences in Reynolds number and diameter ratio primarily affect the number of vortices involved in the interaction, rather than the form of the interaction itself. The greater the number of vortices in any one beat, the more simple the linkage process. As the number of vortices in any one beat becomes small, as for the 1.76 diameter ratio, then the behavior becomes less easily described and far less periodic.

The vortex linkages of the indirect mode are significantly different from the direct mode. The indirect mode, which we suspect to be caused by a propagating disturbance, is a more complex problem. The disturbance, if caused by the velocity shear as we suggest, would contain vertical vorticity. A model of the vortex linkages for the indirect mode would need to include the interaction of the vertical vorticity with the spanwise vorticity.

The models of vortex linkages suggested so far all appear to be appropriate only for the direct mode of interaction. Gerrard's model ${ }^{7}$ is suggested as representing the possible vortex linkages behind a diameter discontinuity or velocity discontinuity, as a simplification of the modulated shedding caused by flow nonuniformity. That is exactly the motivation for our experiments. The modulation caused by flow nonuniformity is more complex, and would be more like the indirect mode.

The model suggested by Williamson ${ }^{7}$ is for the indirect mode, as can be seen from the inclined interfaces of his flow visualization. His model does not incorporate the spanwise propagation that takes place. Our studies suggest that a much more complex process is occurring.

Our experiments on stepped cylinder geometry have helped us to understand some of the features of frequency interaction in wakes, and help to explain some previous observations. For example, consider the geometry used by Eiscnlohr and Eckelmann ${ }^{21}$ as a means to keep vortex shedding parallel to the cylinder. Larger-diameter tubing of at 
least 6 to 7 diameters in length is used as a sleeve over the cylinder model ends. They report that a diameter ratio of between 1.8 and 2.2 is necessary for the method to work. This indicates that the indirect mode is required for control of end effects. In the indirect mode, there is little communication between the coupled vortex streets, because the vortex linkages are mostly in the form of half-loops. This provides, in their terms, the "design break line" necessary for the method to enable parallel vortex shedding of the small-diameter street.

In Gaster's work on cones, it would seem that the frequency modulations of the cone experiment are the result of an indirect mode, since the disturbances are all along the span. Gaster notes that the modulation frequency is the same all along the span of the cone, which indicates that the disturbances are all of the same frequency (perhaps the taper ratio determines the frequency). Gaster's tapered cylinder results, however, show discrete cells along the span of the cylinder. One of two possibilities seems likely. (1) The taper is small enough so that the frequency variation is handled through a series of direct modes. (2) The growth rate of the indirect mode disturbance is small and becomes organized into a series of relative slow growth regions.

In future work, we intend to utilize a forcing technique to create a frequency discontinuity in the wake of a model of constant cross section. Also, we would like to extend these studies to higher Reynolds numbers. In addition, we observed that the modulated region of the direct mode appeared to undergo an earlier transition to turbulence than did neighboring wake regions. We would like to study this phenomenon and its relationship to other flows that exhibit localized regions of early transition, such as turbulent spots in the boundary layer.

\section{ACKNOWLEDGMENTS}

We wish to thank Professor F. Browand for suggesting the use of the wavelet transform in this study and for helpful discussion and comments.

This work was supported by the Ocean Engineering Division of ONR, Grant No. N00014-90-J-1314, and by DARPA/URI Grant No. N00014-86-K-0758.
${ }^{1} \mathrm{M}$. Gaster, "Vortex shedding from slender cones at low Reynolds numbers," J. Fluid Mech. 38, 565 (1969).

${ }^{2} \mathrm{M}$. Gaster, "Vortex shedding from circular cylinders at low Reynolds numbers," J. Fluid Mech. 46, 749 (1971).

${ }^{3}$ D. J. Maull and R. A. Young, "Vortex shedding from bluff bodies in a shear flow," J. Fluid Mech. 60, 401 (1973).

${ }^{4}$ W. A. Mair and P. K. Stansby, "Vortex wakes of bluff cylinders in shear flow," SIAM J. Appl. Math. 28, 519 (1975).

${ }^{5} \mathrm{P} . \mathrm{K}$. Stansby, "The effects of end plates on the base pressure coefficient of a circular cylinder," Aeronaut. J. 78, 36 (1974).

${ }^{6} \mathrm{D}$. Gerich and H. Eckelmann "Influence of end plates and free ends on the shedding frequency of circular cylinders," J. Fluid Mech. 122, 109 (1982).

${ }^{7}$ C. H. K. Williamson, "Oblique and parallel modes of vortex shedding in the wake of a circular cylinder at low Reynolds numbers," J. Fluid Mech. 206, 579 (1989).

${ }^{8} \mathrm{M}$. Konig, H. Eisenlohr, and H. Eckelmann, "The fine structure in the Strouhal-Reynolds number relationship of the laminar wake of a circular cylinder," Phys. Fluids A 2, 1607 (1990).

"J.H. Gerrard, "The three-dimensional structure of the wake of a circular cylinder," J. Fluid Mech. 25, 143 (1966).

${ }^{10}$ F. K. Browand and C. M. Ho, "Forced, unbounded shear flows," Nucl. Phys. B (Suppl.) 2, 139 (1987).

"F. K. Browand and S. Prost-Domasky, New Trends in Nonlinear Dynamics and Pattern Forming Phenomena: The Geometry of Non Equilibrium, edited by P. Coullet and P. Huerre (Plenum, New York, 1990).

${ }^{12}$ T. Dallard and F. K. Browand, "Scale transitions at defect sites in the mixing layer: Application of the 2-D arc wavelet transform," submitted to J. Fluid Mech., 1991.

${ }^{13}$ P. Huerre, "Evolution of coherent structures in shear flows: A phase dynamics approach," Nucl. Phys. B (Suppl.) 2, 159 (1987).

${ }^{14}$ N. W. M. Ko, W. L. Leung, and H Au, "Flow behind two coaxial circular cylinders," J. Fluids Eng. 104, 223 (1982).

${ }^{15}$ N. W. M. Ko and A. S. K. Chan, "In the intermixing region behind circular cylinders with stepwise change of the diameter," Exp. Fluids 9, 213 (1990).

${ }^{16} \mathrm{C}$. Van Atta and M Gharib, "Ordered and chaotic vortex streets behind circular cylinders at low Reynolds numbers," J. Fluid Mech. 174, 113 (1987).

${ }^{17} \mathrm{C}$. Willert and $\mathrm{M}$ Gharib, "A new video technique to capture images generated by a smoke wire," Exp. Fluids 8, 238 (1989).

${ }^{18} \mathrm{M}$. Hammache and M. Gharib, "A novel method to promote parallel vortex shedding in the wake of circular cylinders," Phys. Fluids A 1, 1611 (1989).

${ }^{19}$ Wavelets, edited by J. M. Combes, A. Grossman, and $\mathrm{Ph}$. Tchamitchian (Springer-Verlag, Berlin, 1989).

${ }^{20} \mathrm{~J}$. Liandrat and F. Moret-Bailly, "The wavelet transform: some applications to fluid dynamics and turbulence," Eur. J. Mech. B: Fluids 9, 1 (1990).

${ }^{21} \mathrm{H}$. Eisenlohr and H. Eckelmann, "Vortex splitting and its consequences in the vortex street wake of cylinders at low Reynolds number," Phys. Fluids A 1, 189 (1989). 